\title{
MINIMAL IMMERSIONS OF LOW DIMENSIONAL MANIFOLDS
}

\author{
ROBERT D. LITTLE
}

Abstract. We show that if $M$ is a spin $n$-manifold, $n<7$, then $M$ immerses in $\mathbf{R}^{n+3}$.

1. Introduction. Let $M$ be a connected, smooth $n$-manifold. A theorem of Whitney [5] says that if $n \geqslant 1, M$ immerses in $\mathbf{R}^{2 n-1}$. We show that if $n<7$ and $M$ is a spin manifold, that is, $M$ is closed, orientable and $w_{2}(M)=0$, then $M$ immerses in $\mathbf{R}^{n+3}$. This improves Whitney's theorem in the range of dimensions $5 \leqslant n \leqslant 7$. We also prove two theorems which concern the question of when a map of codimension 1 or 2 is homotopic to an immersion.

We denote the Stiefel-Whitney class of $M$ in $H^{i}\left(M ; \mathbf{Z}_{2}\right)$ by $w_{i}(M)$ and the Pontrjagin class in $H^{4}(M ; Z)$ by $P_{1}(M)$. If $v$ is a class in $H^{2}(M ; Z)$, its reduction mod 2 is denoted by $\bar{v}$ and the Bockstein cohomology operation associated with reduction mod 2 is denoted by $\delta$.

THEOREM 1.1. If $M$ is a spin $n$-manifold and $n \leqslant 7$, then $M$ immerses in $\mathbf{R}^{n+3}$.

THEOREM 1.2. Let $M$ be an $n$-manifold where $n<7$ and let $f: M \rightarrow N$ be $a$ continuous map of codimension 2 such that $f^{*} w_{1}(N)=w_{1}(M)$. If $H^{4}(M ; Z)$ has no elements of order 4 , then $f$ is homotopic to an immersion if and only if there is $a$ class $v$ in $H^{2}(M ; Z)$ such that $w_{i}(M)+w_{i-2}(M) \bar{v}=f^{*} w_{i}(N), i<4$, and $P_{1}(M)+v^{2}=f^{*} P_{1}(N)$.

THEOREM 1.3. Let $M$ be an $n$-manifold where $n<7$ and let $f: M \rightarrow N$ be $a$ continuous map of codimension 1 . If $H^{4}(M ; Z)$ has no elements of order 4 , then $f$ is homotopic to an immersion if and only if there is a class $u$ in $H^{1}\left(M ; Z_{2}\right)$ such that $w_{i}(M)+w_{i-1}(M) u=f^{*} w_{i}(N), i \leqslant 4$, and $P_{1}(M)+\left[\delta w_{1}(M)\right][\delta u]$ $=f^{*} P_{1}(N)$.

Thomas shows that if $n \equiv 3(\bmod 4)$, then any spin $n$-manifold immerses in $\mathbf{R}^{2 n-3}$ [10]. Theorem 1.1 sharpens Thomas' result by one dimension in the case $n=7$. We will give examples which show that Theorem 1.1 is best possible for $n=6,7$. In these two dimensions, the theorem is stronger than the well-known conjecture that every $n$-manifold can be immersed in

Received by the editors July 29, 1975 and, in revised form, December 20, 1976.

AMS (MOS) subject classifications (1970). Primary 55G35, 55G36.

Key words and phrases.Obstructions, functional cohomology operations, independent vector fields, immersions.

O American Mathematical Society 1977 
$\mathbf{R}^{2 n-\alpha(n)}$, where $\alpha(n)$ is the sum of the digits in the binary expansion of $n$. Hirsch shows that for $n=5$, the spin hypothesis is not necessary [1]. Theorems 1.2 and 1.3 are the same as the theorems of Thomas [11], except that the condition $H^{4}(M ; Z)$ has no 2-torsion has been reduced to the assumption that $H^{4}(M ; Z)$ has no elements of order 4 .

2. The proofs of Theorems 1.1, 1.2 and 1.3. The proofs of the above theorems involve the three obstruction formulas stated below. The first formula is due to Olum [8] and the second and third formulas are the main theoretical results of this paper. If $X$ is a complex and $f, g: X \rightarrow Y$ are maps, Olum associates with each class $y$ in $H^{i}(Y ; G)$ a homomorphism $y_{\#}: \pi_{i}(Y) \rightarrow G$ and then shows that $f^{*} y-g^{*} y=y_{*} O^{i}(f, g)$, when the obstruction to a homotopy, $O^{i}(f, g)$, is nonvoid. The homomorphism $y_{\#}$ is the composite of the Hurewicz homomorphism and evaluation. If $\xi$ and $\eta$ are two stable bundles over a complex $X$ with $w_{i}(\xi)=w_{i}(\eta), i \leqslant 2$, then the second obstruction to a stable isomorphism, $\mathrm{O}^{4}(\xi, \eta)$, is a nonvoid subset of $H^{4}(X ; \mathrm{Z})$ and formula (2.2) follows from Olum's formula. (See [8, p. 95].) Formula (2.3) involves the functional cohomology operation associated with the action of $\delta \mathrm{Sq}^{2}$ on the cohomology sequence of the pair $\left(X \times I \cup B O_{F}, B O_{F}\right)$, where $B O_{F}$ is the mapping cylinder of the map $F: X \times \dot{I} \rightarrow B O$ given by the classifying maps of $\xi$ and $\eta$. If $w_{2}(\xi)=w_{2}(\eta)$, this operation is defined on $w_{2}$ in $H^{2}\left(B O ; \mathbf{Z}_{2}\right)$ and the resulting subset of $H^{4}(X ; \mathrm{Z})$ is denoted by $\delta \mathrm{Sq}_{\xi, \eta}^{2}\left(w_{2}\right)$. This subset is a coset modulo $\left(P_{1}(\xi)-P_{1}(\eta)\right)$, the subgroup generated by the difference $P_{1}(\xi)-P_{1}(\eta)$. (See [3] or [7].)

Proposition 2.1. If $\xi$ and $\eta$ are two stable bundles such that $w_{i}(\xi)=w_{i}(\eta)$, $i<2$, then the second obstruction to a stable isomorphism, $O^{4}(\xi, \eta)$, satisfies the equations:

$$
\begin{gathered}
2 O^{4}(\xi, \eta)=P_{1}(\xi)-P_{1}(\eta), \\
O^{4}(\xi, \eta)+\left(P_{1}(\xi)-P_{1}(\eta)\right)=\delta \mathrm{Sq}_{\xi, \eta}^{2}\left(w_{2}\right), \\
\bar{O}^{4}(\xi, \eta)=w_{4}(\xi)-w_{4}(\eta) .
\end{gathered}
$$

Proof. If $K\left(\mathbf{Z}_{2}, 2 ; \mathrm{Z}, 4, \delta \mathrm{Sq}^{2}\right)$ is the total space of the fibration induced by $\delta \mathrm{Sq}^{2}, f^{\prime}: B O \rightarrow K\left(\mathrm{Z}_{2}, 2 ; \mathbf{Z}, 4, \delta \mathrm{Sq}^{2}\right)$ a lifting of $w_{2}$, and $[g]$ in $\pi_{4}(B O)$ a generator, it follows from the Peterson-Stein definition of functional cohomology operation $\left[6\right.$, p. 159] that the set $\left\{f_{\#}^{\prime}[g]: f^{\prime *} \iota=w_{2}\right\}$ can be identified with the functional operation $\delta \mathrm{Sq}_{g}^{2}\left(w_{2}\right)$. (See [3].) Direct computation shows that $\delta \mathrm{Sq}_{g}^{2}\left(w_{2}\right)$ is the nonzero coset mod 2, and so (2.3) follows from the naturality of obstructions, 10.8 in [7], and the naturality of functional operations [7, 14.6]. Formula (2.4) follows from the defining diagram of the operation $\delta \mathrm{Sq}_{\xi, \eta}^{2}\left(w_{2}\right)$, reduced $\bmod 2$, and the fact that $\mathrm{Sq}^{3}$ is zero on 2-dimensional classes.

Theorem 1.1 is true by Whitney's theorem if $n \leqslant 4$. The proof in the cases $5 \leqslant n \leqslant 7$ depends on Hirsch's theorem [1]. In order to state Hirsch's theorem, we introduce some notation and a definition. If $\xi$ is a bundle, let $(\xi)$ 
denote its stable equivalence class. We will say that a stable bundle $(\xi)$ has geometric dimension $\leqslant k$ or $\operatorname{gd}(\xi) \leqslant k$ (for some positive integer $k$ ) if $(\xi)$ contains a $k$-plane bundle. For a smooth manifold $M$, we let $\tau M$ denote the tangent bundle and $\nu M$ the stable normal bundle; i.e. $\nu M=-(\tau M)$. Hirsch's theorem says that a continuous map $f: M \rightarrow N$ of codimension $k$ is homotopic to an immersion if and only if $\operatorname{gd}\left(f^{*}(\tau N)+\nu M\right) \leqslant k$. An immediate corollary of this theorem is the fact that $M$ immerses in Euclidean space with codimension $k$ if and only if gd $\nu M \rightarrow k$. Theorem 1.1 will follow from this corollary and the lemma below.

LEMMA 2.5. If $\xi$ is a stable spin bundle over a spin $n$-manifold, $n \leqslant 7$, then gd $\xi \leqslant 3$ if and only if $w_{4}(\xi)=0$.

Proof. The necessity of the condition is clear. If $w_{4}(\xi)=0$, it follows from (2.2) and (2.4) that there is a class $e$ in $H^{4}(M ; Z)$ such that $P_{1}(\xi)=4 e$ and $2 e \in O^{4}(\xi, *)$. There is a 3-plane bundle over $S^{4}, \hat{\gamma}$, such that $P_{1}(\hat{\gamma})=4 \iota$, where $\iota$ is the fundamental class, and so by $(2.2), O^{4}(\hat{\gamma}, *)=2 \iota$ since $H^{4}\left(S^{4}\right.$; $Z$ ) is torsion free. Since $\mathrm{Sq}^{2} e=0$, and $0 \in \Phi(e)$, where $\Phi$ is the secondary operation associated with the relation $\mathrm{Sq}^{2} \mathrm{Sq}^{2}=0(\mathrm{Z})$, as in $[12$, proof of Theorem 5b], we see that by [12, Lemma 5] there is a map $g: M \rightarrow S^{4}$ such that $g^{*} \iota=e$. Thus $\gamma=g^{*} \hat{\gamma}$ is a spin 3-bundle satisfying the conditions $P_{1}(\gamma)=4 e$ and $2 e \in O^{4}(\gamma, *)$. Therefore $\xi$ is stably isomorphic to $\gamma$ since $O^{4}(\xi, \gamma)=O^{4}(\xi, *)-O^{4}(\gamma, *)$ and $\pi_{i}(B O)=0,5 \leqslant i \leqslant 7$, and hence gd $\xi \leqslant 3$.

Theorem 1.1 in the cases $5 \leqslant n \leqslant 7$ now follows from Hirsch's theorem and Lemma 2.5. It is a consequence of Wu's formula that if $M$ is a spin $n$-manifold, $n \leqslant 7$, then $w_{4}(M)=0$ [12]. This means that $\nu M$ is a stable spin bundle such that $w_{4}(\nu M)=0$ and so gd $\nu M \leqslant 3$ by Lemma 2.5 .

Theorems 1.2 and 1.3 follow immediately from Hirsch's theorem, the standard formulas for the characteristic classes of the sum of two bundles, the classification of 1-bundles and orientable 2-bundles in terms of cohomology classes, and the fact that $O^{4}(\xi, \eta)=0$ when $w_{i}(\xi)=w_{i}(\eta), i \leqslant 4, P_{1}(\xi)=$ $P_{1}(\eta)$ and $H^{4}(M ; Z)$ has no elements of order 4. This last fact follows immediately from the obstruction formulas. In both theorems we use Thomas' result on the difference between the Pontrjagin class of the sum of two bundles and the product of the Pontrjagin classes. (See [9].)

It is clear that Theorem 1.1 in the cases $n=6,7$ is the strongest such general theorem that can be proved in the sense that there exist spin $n$-manifolds, $n=6,7$, which do not immerse with codimension 2 . It follows easily from Theorem 1.2 that $\mathbf{C P}^{3}$ is a spin 6-manifold which does not immerse in $\mathbf{R}^{8}$ and that the lens space $L^{7}(3)$ is a spin 7-manifold which does not immerse in $\mathbf{R}^{9}$. Theorem 1.3 clearly produces two immersions due to Hirsch [1]: $\mathbf{R} P^{n}$ immerses in $\mathbf{R}^{n+1}, n=6,7$.

The obstruction formulas of Proposition 2.1 are generalized in [4]. In this paper, we prove a stronger version of Lemma 2.5 and use it to give a proof of 
Hirsch's result that every closed, orientable 5-manifold immerses in $\mathbf{R}^{\mathbf{8}}$.

If $M$ is a manifold, span $M$ is defined to be the maximal number of linearly independent cross-sections of $\tau M$. The two theorems below follow immediately from Lemma 2.5 , Wu's formula and standard bundle theory, [2, 1.7] and [12, Lemma 2]. The first theorem improves a theorem of Thomas: it removes Condition $\mathrm{T}$ from [12, Theorem $5 \mathrm{~b}]$. In the second theorem, $\chi(M)$ denotes the Euler characteristic of $M$. This result was proved by Thomas [12] using different methods.

THEOREM 2.6. If $M$ is a spin 7-manifold, then $\operatorname{span} M>4$.

THEOREM 2.7. If $M$ is a spin 6-manifold, then span $M>3$ if and only if $\chi(M)=0$.

\section{REFERENCES}

1. M. Hirsch, Immersions of manifolds, Trans. Amer. Math. Soc. 93 (1959), 242-276.

2. I. M. James and E. Thomas, An approach to the emumeration problem for non-stable vector bundles, J. Math. Mech. 14 (1965), 485-506.

3. R. D. Little, A relation between obstructions and functional cohomology operations, Proc. Amer. Math. Soc. 49 (1975), 475-480.

4. 327-332. , Torsion elements and the classification of vector bundles, Canad. J. Math. 29 (1977),

5. J. Milnor, Lectures on characteristic classes, mimeographed notes, Princeton Univ., Princeton, N. J., 1957.

6. R. E. Mosher and M. C. Tangora, Cohomology operations and applications in homotopy theory, Harper and Row, New York, 1968.

7. P. Olum, Invariants for effective homotopy classification and extension of mappings, Mem. Amer. Math. Soc. No. 37 (1961).

8. __ Factorizations and induced homomorphisms, Advances in Math. 3 (1969), 72-100.

9. E. Thomas, The torsion Pontrjagin classes, Proc. Amer. Math. Soc. 13 (1962), 485-488.

10. __ Real and complex vector fields on manifolds, J. Math Mech. 16 (1967), 1183-1206.

11. Submersions and immersions with codimension one or two, Proc. Amer. Math. Soc. 19 (1968), 859-863.

12. __ Vector fields on low dimensional manifolds, Math. Z. 108 (1968), 85-93.

Department of Mathematics, University of Hawail, Honolulu, Hawail 96822 